\title{
Enhancing Adaptive Hypermedia Presentation Systems by Lifelike Synthetic Characters
}

\author{
Elisabeth André \\ DFKI GmbH, Stuhlsatzenhausweg 3, D-66123 Saarbrücken, Germany, \\ andre@dfki.de
}

Rapid growth of competition in the electronic market place will boost the demand for new innovative communication styles to attract web users. With the advent of web browsers that are able to execute programs embedded in web pages, the use of animated characters for the presentation of information over the web has become possible. Instead of surfing the web on their own, users can join a tour, ask the lifelike character for assistance or even delegate a complex search task to it.

Over the past few years, we developed a number of personalized information assistants that facilitate user access to the Web [2] by providing orientation assistance in a dynamically expanding navigation space. These assistants are characterized by their ability to retrieve relevant information, reorganize it, encode it in different media (such as text, graphics, and animation), and present it to the user as a multimedia presentation.

The screen shot in Fig. 1 shows one of our applications, which is a personalized travel agent. Suppose the user wants to travel to Hamburg and is starting a query for typical travelling information. To comply with the user's request, the system retrieves information about Hamburg from various web servers, e.g. a weather, a restaurant and a hotel server, selects relevant units, restructures them and uses an animated character to present them to the user. The novelty of our approach is that the presentation scripts for the character and the hyperlinks between the single presentation parts are not stored in advance but generated automatically from pre-authored documents fragments and items stored in a knowledge base. For a restricted domain, we are even able to combine information units retrieved from different sources and combine them into a single presentation item. For example, the address entry of a hotel is used as input for another web search in order to generate a map display on which the hotel can be located.

Though a number of similarities may exist, our presentation agents are not just animated icons in the interface. Rather, their behavior follows the equation:

\section{Persona behavior $:=$ directives + self-behavior}

By directives we understand a set of tasks which can be forwarded to a character for execution. To accomplish these tasks, the character relies on gestures that: express 
emotions (e.g., approval or disapproval), convey the communicative function of a presentation act (e.g., warn, recommend or dissuade), support referential acts (e.g., look at an object and point at it), regulate the interaction between the character and the user (e.g., establishing eye contact with the user during communication) and indicate that the character is speaking. We use the term presentation script to refer to a temporally ordered set of directives.

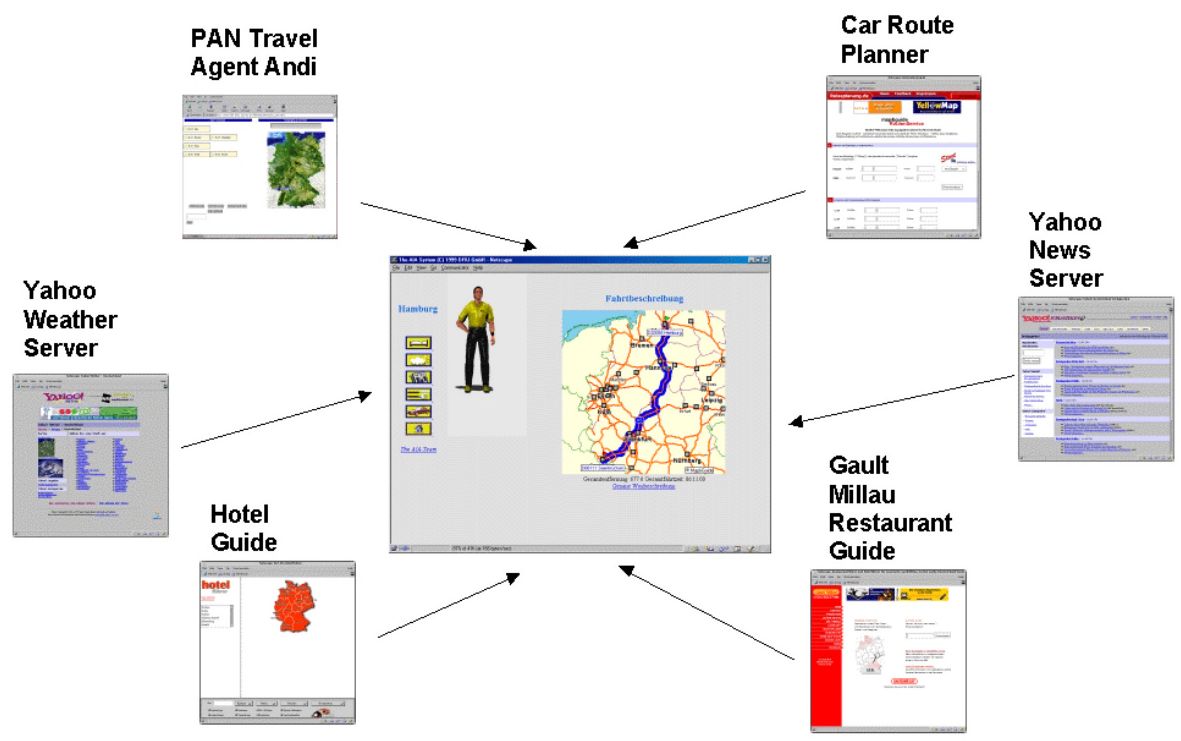

Fig. 1. Personalized Travel Agent.

While a script is an external behavior determinant that is specified outside the character, our characters also have an internal behavior determinant resulting in what we call a self behavior. A character's self behavior comprises not only gestures that are necessary to execute the script, but also navigation acts, idle time gestures, and immediate reactions to events occurring in the user interface.

Since the manual scripting of agent behaviors is tedious, error-prone and for timecritical applications often unfeasible, we aimed at the automation of the authoring approach. Based on our previous work on multimedia presentation design [1], we utilize a hierarchical planner for the automated decomposition of high-level presentation tasks into scripts which will be executed by the presentation agent [2]. To flexibly tailor presentations to the specific needs of an individual user, we allow for the specification of generation parameters (e.g., "verbal utterances should be in English", or "the presentation must not exceed five minutes"). Consequently a number of presentation variants can be generated for one and the same piece of 
information, but different settings of presentation parameters. Furthermore, we allow the user to flexibly choose between different navigation paths through a presentation. That is, the course of a presentation changes at runtime depending on user interactions.

To facilitate the integration of animated agents into web interfaces, DFKI has developed a toolkit called PET (Persona-Enabling Toolkit). PET provides an XMLbased language for the specification of Persona commands within conventional HTML-pages. These extended HTML-pages are then automatically transformed into a down-loadable Java-based runtime environment which drives the presentation on standard web browsers. PET may be used in two different ways. First of all, it can be used by a human author for the production of multimedia presentations which include a lifelike character. Second, we have the option to automate the complete authoring process by making use of our presentation planning component to generate web pages that include the necessary PET-commands.

In the talk, the approach will be illustrated by means of several academic and industrial projects currently being carried out at DFKI GmbH.

Part of this research was supported by the German Ministry for Education, Science, Research and Technology (BMBF) under contract 01 IW 806 and by the European Community under the contracts ERB 4061 PL 97-0808 and EP-29335. The talk is based on work by the following people (in alphabetical order): Steve Allen, Elisabeth André, Stephan Baldes, Patrick Gebhard, Bernhard Kirsch, Thomas Kleinbauer, Martin Klesen, Jochen Müller, Susanne van Mulken, Stefan Neurohr, Peter Rist, Thomas Rist, Ralph Schäfer and Wolfgang Wahlster.

\section{References}

1. André, E., and Rist, T. (1995). Generating coherent presentations employing textual and visual material. Artificial Intelligence Review, Special Issue on the Integration of Natural Language and Vision Processing 9(2-3):147-165.

2. André, E., Rist, T. and Müller, J. (1999). Employing AI Methods to Control the Behavior of Animated Interface Agents. Applied Artificial Intelligence 13:415-448.

3. André, E., Rist, T. and Müller, J. (1998). Guiding the User through Dynamically Generated Hypermedia Presentations with a Life-Like Presentation Agent. In: Proceedings of the 1998 International Conference on Intelligent User Interfaces, pp. 21-28, New York: ACM Press.

4. André, E., Rist, T. and Müller, J. (1998). Integrating Reactive and Scripted Behaviors in a Life-Like Presentation Agent. In: Proceedings of the Second International Conference on Autonomous Agents (Agents '98), pp. 261-268, New York: ACM Press.

5. André, E. Rist, T. and J. Müller (1998). WebPersona: A Life-Like Presentation Agent for the World-Wide Web. Knowledge-Based Systems, 11(1):25-36, 1998.

6. André, E. and Rist, T. (2000). Presenting through Performing: On the Use of Multiple Life-Like Characters in Knowledge-Based Presentation Systems. In: Proceedings of the 1998 International Conference on Intelligent User Interfaces, pp. 1-8, New York: ACM Press.

7. André, E., Rist, T. , van Mulken, S., Klesen, M. and Baldes, S. (2000). The Automated Design of Believable Dialogues for Animated Presentation Teams. In: Cassell et al. (eds.): Embodied Conversational Agents, 220-255, Cambridge, MA: MIT Press. 
8. van Mulken, S., André, E. and Müller, J. (1998). The Persona Effect: How Substantial is it? In: Proc. of HCI'98, Sheffield, pp. 53-66.

9. van Mulken, S., André, E. and Müller, J. (1999). An empirical study on the trustworthiness of lifelike interface agents. In H.-J. Bullinger and J. Ziegler, eds., HumanComputer Interaction (Proc. of HCI-International 1999), 152-156. Mahwah, New Jersey: Lawrence Erlbaum Associates. 